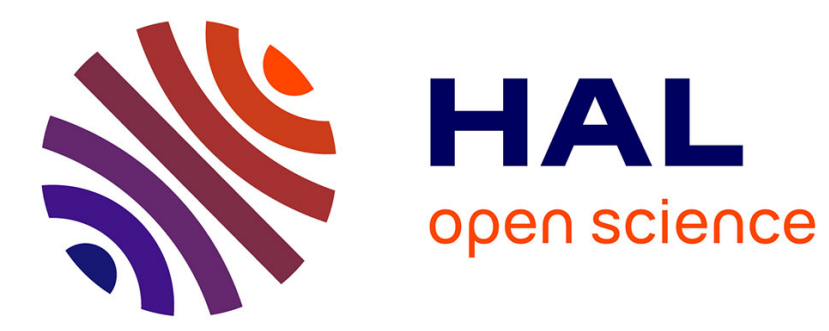

\title{
Large-Scale Fabrication of Surface Ion Traps on a 300 mm Glass Wafer
}

Jing Tao, Jean-Pierre Likforman, Peng Zhao, Hong Yu Li, Theo Henner, Yu Dian Lim, Wen Wei Seit, Luca Guidoni, Chuan Seng Tan

\section{- To cite this version:}

Jing Tao, Jean-Pierre Likforman, Peng Zhao, Hong Yu Li, Theo Henner, et al.. Large-Scale Fabrication of Surface Ion Traps on a $300 \mathrm{~mm}$ Glass Wafer. physica status solidi (b), 2021, 258, 10.1002/pssb.202000589 . hal-03455800

\section{HAL Id: hal-03455800 \\ https://hal.science/hal-03455800}

Submitted on 29 Nov 2021

HAL is a multi-disciplinary open access archive for the deposit and dissemination of scientific research documents, whether they are published or not. The documents may come from teaching and research institutions in France or abroad, or from public or private research centers.
L'archive ouverte pluridisciplinaire HAL, est destinée au dépôt et à la diffusion de documents scientifiques de niveau recherche, publiés ou non, émanant des établissements d'enseignement et de recherche français ou étrangers, des laboratoires publics ou privés. 


\title{
Large-Scale Fabrication of Surface Ion Traps on a $300 \mathrm{~mm}$ Glass Wafer
}

\author{
Jing Tao, * Jean-Pierre Likforman, Peng Zhao, Hong Yu Li, Theo Henner, Yu Dian Lim, \\ Wen Wei Seit, Luca Guidoni,* and Chuan Seng Tan*
}

Herein, the large-scale fabrication of radio frequency (RF) surface ion traps on a $300 \mathrm{~mm}$ glass wafer using a standard foundry process is reported. Established wafer-level packaging process of electroplated $\mathrm{Cu}$ with $\mathrm{Au}$ finish is used to fabricate the surface electrodes directly on the glass wafer substrate. A trap is tested by loading it with laser-cooled ${ }^{88} \mathrm{Sr}^{+}$ions. The trap shows a stable operation with RF amplitudes in the range of 100-250 V at $33 \mathrm{MHz}$ frequency. The ion lifetime is on the order of $\mathbf{3 0}$ min with laser cooling with a vacuum chamber pressure of $\sim 5 \times 10^{-11}$ mbar. These results demonstrate the potential of large-size foundry glass substrates to realize scalable and integratable trapped ion-based quantum devices.

\section{Introduction}

In the past decades, trapped ion technologies have been rapidly developed for applications in quantum computing, ${ }^{[1-3]}$ simulation, ${ }^{[4,5]}$ and sensing. ${ }^{[6,7]}$ Invented in $2005^{[8]}$ and demonstrated for the first time in $2006,{ }^{[9]}$ the surface ion trap geometry, in which all the electrodes lie in the same plane, enables microfabrication flexibility for complex electrode designs, ${ }^{[10,11]}$ optoelectronic integration ${ }^{[12,13]}$ enhancing then trap scalability and functionality. Trapped ions are among the most promising systems to realize scalable quantum computers due to their capability in precise manipulation of multiple ion qubits with high fidelity ${ }^{[14]}$ and long coherence time. ${ }^{[15]}$ Some challenges still affect the implementation of these systems including the use of standard foundry processes on a low radio frequency (RF)-loss

Dr. J. Tao, P. Zhao, Dr. Y. D. Lim, Prof. C. S. Tan

School of Electrical and Electronic Engineering

Nanyang Technological University

Singapore 639798, Singapore

E-mail: taojing@ntu.edu.sg; tancs@ntu.edu.sg

Dr. J.-P. Likforman, T. Henner, Dr. L. Guidoni

Laboratoire Matériaux et Phénomènes Quantiques

Université Paris Diderot

Paris F-75025, France

E-mail: luca.guidoni@univ-paris-diderot.fr

Dr. H. Y. Li, W. W. Seit

Institute of Microelectronics

Agency for Science, Technology and Research (A*STAR)

Singapore 117685, Singapore

The ORCID identification number(s) for the author(s) of this article can be found under https://doi.org/10.1002/pssb.202000589.

DOI: 10.1002/pssb.202000589 substrate for future high complexity trap fabrication. Quartz, ${ }^{[9]}$ sapphire, ${ }^{[16]}$ and printed-circuit board ${ }^{[17]}$ have been used as low-loss trap substrates. However, the customized fabrication processes with these dielectric substrates pose the difficulties for mass foundry production and process integration. Silicon, as the standard foundry substrate, has been developed as mature surface trap substrate since 2010. ${ }^{[18]}$ Many electrical and optical components, e.g., trench capacitor, ${ }^{[19]}$ digital-toanalog converter (DACs), ${ }^{[20]}$ waveguide, ${ }^{[21]}$ grating coupler, ${ }^{[21]}$ and photon detectors, ${ }^{[22]}$ have been monolithically integrated into $\mathrm{Si}$ traps. However, standard Si substrates have high RF loss tangent, which need proper RF shielding design ${ }^{[18,19,23]}$ and a thick layer of dielectric (up to $10 \mu \mathrm{m}$ ) as insulator to achieve a low trap capacitance. ${ }^{[18,19]}$ These features add complexities and costs for trap fabrication. In this work, we demonstrate the potential of industrial glass substrates available as standard foundry wafer allowing for fabrication sizes up to $300 \mathrm{~mm}$. In particular, these large wafers allow us to exploit the whole toolbox of high-resolution processes that only operate on large-size microfabrication.

Because of its superior dielectric properties, glass could represent a low-cost substitutional material to quartz or sapphire for surface ion trap fabrication. Compared with high resistivity $(\approx 5000 \Omega \mathrm{cm})$ Si substrate with a typical loss tangent of 1.5 at $20 \mathrm{MHz},{ }^{[24]}$ the glass substrate (Corning SGW 8.5) that is adopted in this work has a loss tangent of 0.025 at $5 \mathrm{GHz}$ with volume resistivity of $\approx 10^{10} \Omega \mathrm{cm}$ (data available from product information sheet). This eliminates the need of RF shielding and insulation layers that are required for Si traps and permits much simpler fabrication procedures. In addition, the transparent glass $(\approx 90 \%$ transmission for wavelength from 300 to $2400 \mathrm{~nm}$ ) can make light delivery and collection more flexible, for example, by placing optical fibers and/or photodetectors underneath. ${ }^{[25]}$ Compared with other dielectric material (e.g., sapphire and quartz), glass features not only low cost but also more advanced manufacturability, allowing the implementation of high-reliability through-glass-vias, ${ }^{[26,27]}$ anodic bonding, ${ }^{[28]}$ and also enabling optical integration and coupling to various optical components. ${ }^{[29,30]}$ These techniques provide glass comparable electrical and optical routing capability to silicon which may address the scalability issues of surface trap design and fabrication toward the integration of photonic electronic and detection components. $^{[31]}$ 


\section{Trap Design and Fabrication}

In this work, trap fabrication is based on $300 \mathrm{~mm}$ glass wafer platform with a standard foundry process, as shown in Figure 1a. Traps adopt a linear 5 -wire geometry ${ }^{[8]}$ with RF line widths of $20,40,80$, and $120 \mu \mathrm{m}$. As an example, the trap shown in Figure $1 \mathrm{~b}$ is an $80 \mu \mathrm{m}$ trap with a nominal ion-surface distance $d=75 \mu \mathrm{m}$. The DC and RF electrodes are designed to provide ion confinement in axial $(z)$ and radial $(x-\gamma)$ directions; typical RF voltages with amplitudes of $100-250 \mathrm{~V}$ and frequency of 30-40 MHz are applied to the two "RF" electrodes, whereas static $\mathrm{DC}$ voltages in the range from -10 to $10 \mathrm{~V}$ are used to drive the central control electrode marked as "DC," the two lateral control electrodes marked as "COMP," and the four endcaps marked as “EC." With typical trap chip sizes of $64 \mathrm{~mm}^{2}$ or less, $\approx 1600$ ion traps are fabricated simultaneously in a single wafer. To fabricate the trap electrodes, a Ti/Cu seed layer of $\approx 200 \mathrm{~nm}$ thickness is physically vapor deposited on a glass substrate, followed by mask-defined electroplating $\mathrm{Cu}$ of $\approx 3000 \mathrm{~nm}$ thickness and subsequent electroplating $\mathrm{Au}$ of $\approx 200 \mathrm{~nm}$ thickness as a protective layer. The schematic trap layer information is shown in Figure 1c. The final aspect ratio of electrode thickness to interelectrode gap is $\approx 1$. Due to the relatively thick Au finish layer of $\approx 200 \mathrm{~nm}$, the $\mathrm{Cu}$ electrodes are fully covered with Au. One process issue with $\mathrm{Cu} / \mathrm{Au}$ metal electroplating is the presence of an Au overhang structure which possibly arises during $\mathrm{Cu}$ seed wet etching process, leading to high leakage current between the small-gap electrodes. To resolve this, process optimization has been carried out by reducing the wet etching time to reduce the shortage probability. ${ }^{[32]}$ By conducting $I-V$ test between any DC/RF electrodes, the measured leakage current at $200 \mathrm{~V}$ voltage bias is $\approx 10^{-8} \mathrm{~A}$ in atmospheric condition $\left(22-23^{\circ} \mathrm{C}\right.$, $<60 \% \mathrm{RH})$. The optimized foundry process shows a good yield of short-free traps: the whole set of ten randomly selected traps from one fabricated wafer passed the maximum leakage current limit set of $10^{-8} \mathrm{~A}$. After wafer fabrication, the individual trap die is diced and packaged in a 121-pin ceramic pin grid array (CPGA) package (Kyocera) with a high-vacuum compatible die attach paste (EPO-TEK H20E) and $25 \mu \mathrm{m}$ diameter Au wire bonding to make electrical connections. The optical image of an ion trap in the CPGA package is shown in Figure 1d. Thin single layer ceramic capacitors of $820 \pm 20 \% \mathrm{pF}$ (ATC, 116UL821M100TT) are used to filter RF pickup noise on DC electrodes. ${ }^{[33]}$

\section{Substrate Effect on RF Dissipation}

RF dissipation is one important issue in ion trap design. In the previous finite element study, it shows that the surface ion trap based on glass substrate has lower stray E-field, lower insertion loss, and higher $Q$ factor compared with its Si counterparts. ${ }^{[34]}$ In our experiment, the RF voltage required for ion trap operation (amplitude in the range of 100-250 V) is obtained through a resonant transformer circuit driven by a $50 \Omega \mathrm{RF}$ amplifier. An ideal trap should perform as a pure capacitive load in the $\mathrm{pF}$ range with negligible equivalent series resistance (ESR). Si is a known high-loss material due to its much lower resistivity compared with glass, which induces non-negligible ESR and large capacitance through metal-insulator-silicon structure and $\mathrm{Si}$ substrate itself. ${ }^{[35]}$ In contrast, glass substrate can be regarded (a)

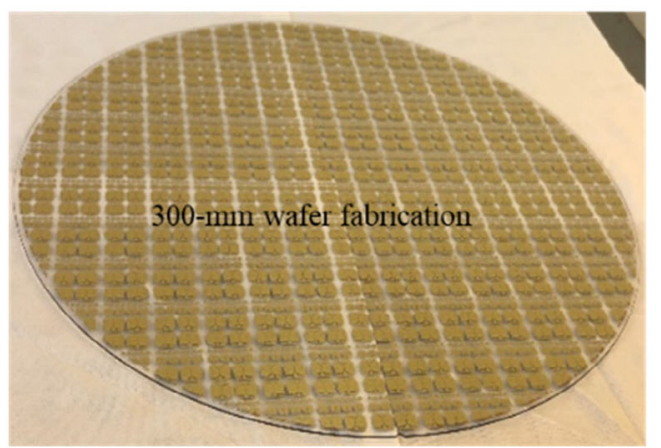

(c)

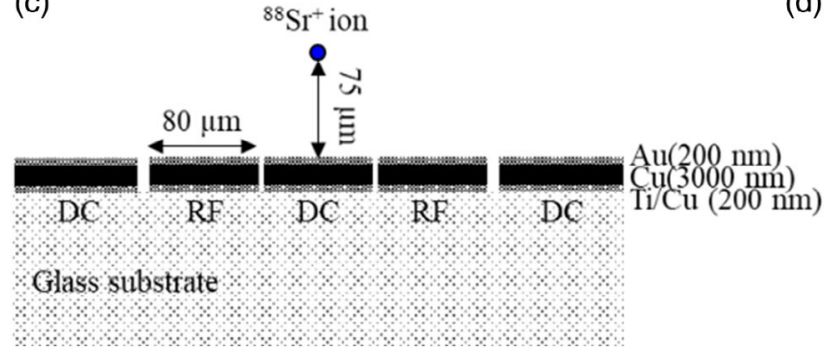

(b)

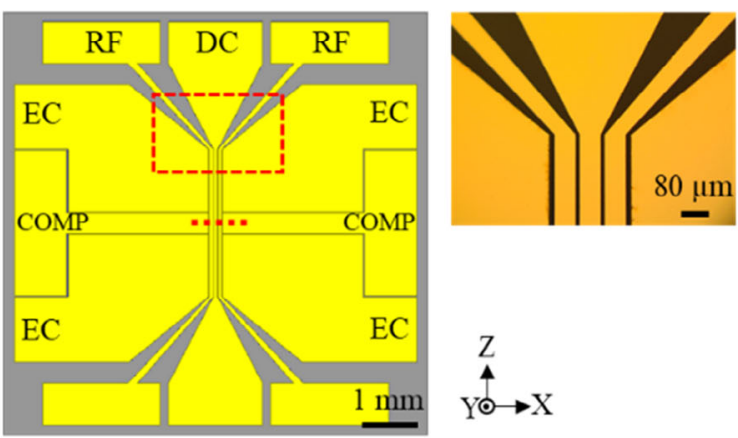

(d)

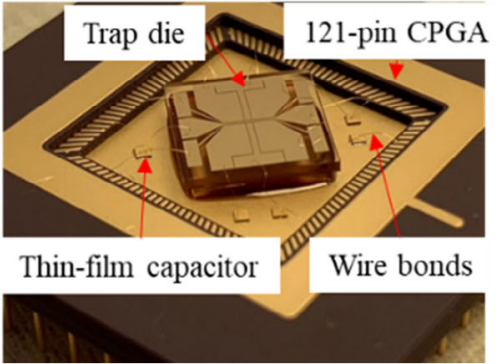

Figure 1. a) Trap die fabricated on a $300 \mathrm{~mm}$ glass wafer. b) Schematic of trap geometry. The RF line width is $80 \mu \mathrm{m}$ and the electrode gap is $5 \mu \mathrm{m}$. The names of individual electrodes are marked accordingly. The optical image of the surface trap corresponding to the marked square area in the schematic is show on the right. c) The cross-sectional schematic corresponding to the dotted mark line in (b) of the surface trap 5-wire geometry with RF width, ion distance and the electrode metal structure marked in the drawing. The dimensions in the schematic are not drawn to scale. d) A trap die packaged in 121-pin CPGA with wire-bonding connections and thin-film capacitors as low-pass filters. 
Table 1. Material properties of $\mathrm{Si}$ and glass substrate for surface trap fabrication. ${ }^{[8]}$ Values are given for readily available substrates at room temperature $\left(25^{\circ} \mathrm{C}\right)$ for frequencies in the RF range.

\begin{tabular}{lcccccc}
\hline $\begin{array}{l}\text { Substrate } \\
\text { material }\end{array}$ & $\begin{array}{c}\text { Wafer } \\
\text { size } \\
{[\mathrm{mm}]}\end{array}$ & $\begin{array}{c}\text { Thermal } \\
\text { conductivity } \\
{\left[\mathrm{W}(\mathrm{m} \mathrm{K})^{-1}\right]}\end{array}$ & $\begin{array}{c}\text { Electrical } \\
\text { resistivity } \\
{[\Omega \mathrm{cm}]}\end{array}$ & $\begin{array}{c}\text { Dielectric } \\
\text { constant }\end{array}$ & $\begin{array}{c}\text { Loss } \\
\text { tangent }\end{array}$ & $\begin{array}{c}\text { Dielectric } \\
\text { strength } \\
{\left[\mathrm{kV} \mathrm{mm}^{-1}\right]}\end{array}$ \\
\hline $\mathrm{Si}$ & 300 & 150 & 5 & 11.7 & 1.5 & 30 \\
glass & 300 & 1.07 & $10^{10}$ & 7.2 & 0.025 & 41 \\
\hline
\end{tabular}

as a nearly lossless substrate with very high resistivity and small loss tangent. The relevant material properties of the two substrates are shown in Table 1. With the given electrical properties (relative permittivity, electrical conductivity, loss tangent) in Table 1, we simulate $S$-parameter of $\mathrm{Si}$ and glass trap by building the respective trap model in COMSOL, as shown in Figure 2a, and configuring lumped ports model on the respective RF-DCRF lines of the trap model for electrical wave incident on port 1 and port 2. The simulated $S$-parameters plots are shown in Figure 2b. Clearly, glass trap shows significantly lower insertion (S21) and reflection (S11) losses as compared with Si trap. Furthermore, we calculate the power loss using Equation (1)

$\operatorname{Loss}(\omega)=1-\frac{S_{21}(\omega) S_{21}^{*}(\omega)}{1-S_{11}(\omega) S_{11}^{*}(\omega)}$
Figure 2c shows the calculated power loss of $\mathrm{Si}$ and glass traps as a function of frequency. The results indicate up to $1.2 \mathrm{~dB}$ $(\approx 23.6 \%)$ power loss for Si substrate in the trapping frequency of $10-100 \mathrm{MHz}$ as compared with only up to $0.04 \mathrm{~dB}(\approx 0.1 \%)$ power loss for glass substrate in the same frequency range. In the trapping experiment, we evaluated trap dissipation by conducting a $\mathrm{RF}$ resonator test for $\mathrm{Si}^{[32]}$ and glass traps fabricated with the same geometry. The testing circuit is similar to the one used in the trapping experiments with a resonant transformer connected to one of the RF and DC lines of the packaged traps through CPGAs pins. The circuit model is shown in Figure 3a which is a series RLC (resistor-inductor-capacitor) resonant circuit driven by an AC voltage source. The parasitic components induced by the trap are represented by the trap capacitance, $C_{\text {trap }}$ and an ESR. We carried out comparative tests between the glass trap, the silicon trap, an ultralow ESR capacitance (AVX SQ series capacitor, $5.6 \mathrm{pF}, \mathrm{ESR}$ in the $\mathrm{m} \Omega$ range) wire-bonded to another CPGA to retrieve from a fit of the trap resonance curves the ESRs associated to the traps and then the ratio between the RF losses of the two traps. The measured resonance curves (Figure $3 \mathrm{~b}$ ) corresponding to the CPGA alone (red curve) and to an ultralow ESR test capacitor of $5.6 \mathrm{pF}$ wire bonded to another CPGA (blue curve) allow us to characterize the resonant circuit and the cabling. Then we measure the resonance curves of the glass and silicon traps (green and orange curves) and we can extract from the fit the ESR of the traps $(54 \mathrm{~m} \Omega$ (a)

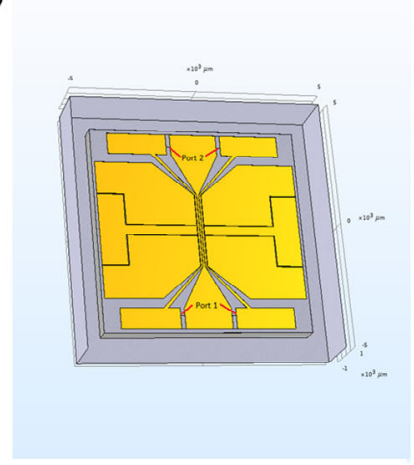

(b)

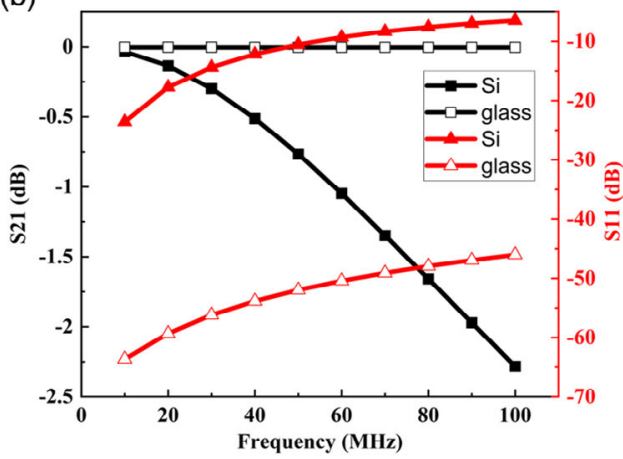

(c)

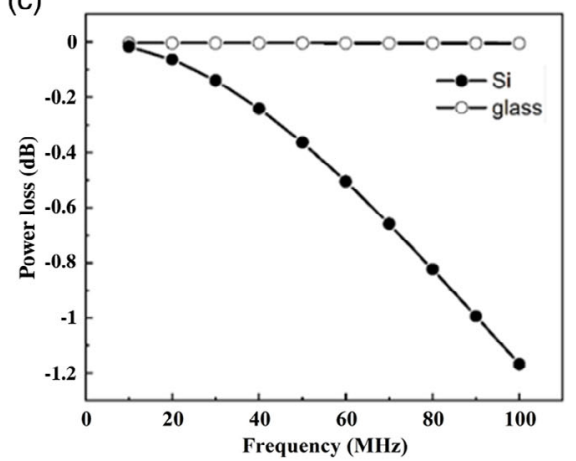

Figure 2. a) Trap model built in COMSOL with lumped port simulation of S-parameters, b) simulated S-parameter curves, S11 and S21, of Si and glass traps, respectively, c) the calculated power loss as a function of frequency for Si and glass traps, respectively.

(a)

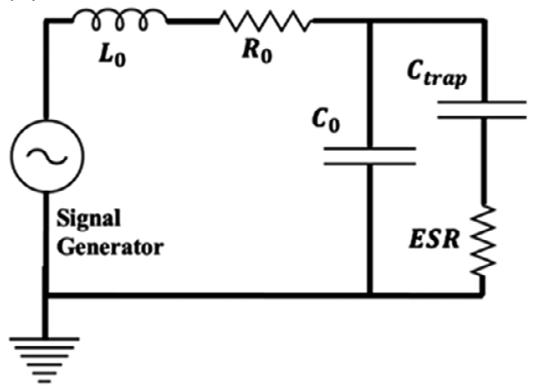

(b)

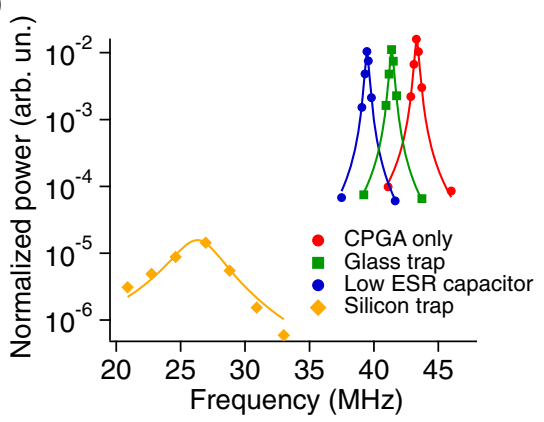

Figure 3. a) Ciruict model of resonator circuit under testing, $C_{\text {trap }}$ and ESR are the trap induced parastic capacitance and resistance, b) measured RF resonance curves of CPGA alone, test capacitor bounded to CPGA (low ESR test capacitor AVX SQ series, $5.6 \mathrm{pF}$ ), and the glass and Si traps wire-bonded to CPGA. 
and $28 \Omega$ for glass and Si traps, respectively) and their capacitances (2.6 and $47 \mathrm{pF}$ for glass and silicon traps, respectively). With these data, we evaluate the ratio between the RF losses (proportional to the ESR and to $\Omega_{\mathrm{RF}}{ }^{2} \times C^{2}$ of the two traps: a glass trap dissipates 60000 times less than a silicon trap of same geometry.

\section{Ion Trap System Setup}

The packaged trap is inserted in a stainless-steel ultrahigh vacuum (UHV) cylindrical chamber (K.J. Lesker) with a set of viewports and flanges for photon detection, laser input/output, lateral imaging, pumping, and electrical feedthrough. During trap operation, the chamber is pumped by an ion pump (IP) and a titanium sublimator pump (TSP). After the baking and the switching to IP + TSP pumping the residual pressure was in the range of $5 \times 10^{-11}$ mbar. This demonstrates the UHV compatibility of the materials used for fabrication and packaging. Adjustable DC voltages are generated by a computer-controlled DAC card (Measurement Computing PCI-DAC6703), filtered by low-pass passive inductor-capacitor-resistor filters and then steered to CPGA. RF voltage (frequency of $32.7 \mathrm{MHz}$ ) is supplied by a Rigol DG4162 generator, amplified in a $10 \mathrm{~W}, 50 \Omega$ amplifier (DeltaRF LA0005-10) and then adapted to the high impedance of the trap by a toroidal resonant transformer (step-up of 9). With this setup the maximum RF voltage amplitude at the trap is around $250 \mathrm{~V}$.

An atomic beam of neutral $\mathrm{Sr}$ is generated by sublimation of a small Sr dendrite (Aldrich) inserted in a helicoidally tungsten filament. To create $\mathrm{Sr}^{+}$ions the atoms are photo-ionized using a two-color CW (continuous wave) technique. ${ }^{[36,37]}$ In typical experimental conditions, the loading time for an ion is roughly $10 \mathrm{~s}$.

The ${ }^{88} \mathrm{Sr}^{+}$ions are Doppler-cooled addressing the $5^{2} \mathrm{~S}_{1 / 2} \rightarrow 5^{2} \mathrm{P}_{1 / 2}$ transition (711 THz, $\left.422 \mathrm{~nm}\right)$, as shown in Figure 4, with a laser beam (Toptica DL-100 laser diode). To avoid optical pumping into the metastable $4^{2} \mathrm{D}_{3 / 2}$ state, we use two additional lasers ("repumpers") addressing the $299 \mathrm{THz}$ $4^{2} \mathrm{D}_{3 / 2} \rightarrow 5^{2} \mathrm{P}_{3 / 2}$ transition (1003 $\mathrm{nm}$, Toptica DL pro laser diode) and the $290 \mathrm{THz} \quad 4^{2} \mathrm{D}_{5 / 2} \rightarrow 5^{2} \mathrm{P}_{3 / 2}$ transition (1033 nm NKT Koheras Adjustik fiber laser). With this scheme, ${ }^{[38]}$ it is possible to eliminate coherent population trapping issues that may affect Doppler cooling using the repumping scheme based on the driving of the $275 \mathrm{THz} 4^{2} \mathrm{D}_{3 / 2} \rightarrow 5^{2} \mathrm{P}_{1 / 2}$ transition. ${ }^{[39]}$ Nevertheless, we used a $275 \mathrm{THz}$ "readout" laser $(1092 \mathrm{~nm}$ NKT Koheras Adjustik fiber laser) to measure the collection efficiency of the

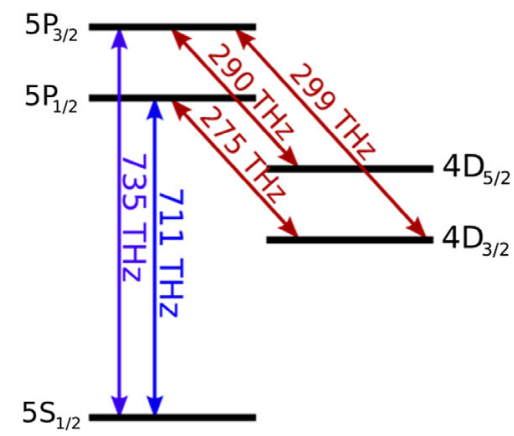

Figure 4. Low lying energy levels of $\mathrm{Sr}^{+}$ion for Doppler cooling. detection system. Frequency stabilization of the $711 \mathrm{THz}$ cooling beam is obtained by a saturated absorption technique in a Rb cell, taking advantage of the near coincidence of the $5^{2} \mathrm{~S}_{1 / 2} \rightarrow 5^{2} \mathrm{P}_{1 / 2}$ transition in ${ }^{88} \mathrm{Sr}^{+}$with the $5^{2} \mathrm{~S}_{1 / 2} \rightarrow 6^{2} \mathrm{P}_{1 / 2}$ transition of neutral ${ }^{85} \mathrm{Rb} .{ }^{[40]}$ Infrared lasers are stabilized with a transfer lock scheme. ${ }^{[41]}$ The frequency gap is filled up and fine tuning of the cooling beam frequency is obtained using a double-pass acousto-optic modulator (AOM). The beam is coupled in a single mode optical fiber and then focused at the ion position with typical power in the $2-50 \mu \mathrm{W}$ range. Repumper lasers are both coupled in a single mode fiber, made collinear with the cooling beam and focused at the ion position with a typical power of $1 \mathrm{~mW}$ per beam.

About $711 \mathrm{THz}$ photons scattered by the ion are collected by a homemade objective with numerical aperture of 0.4 , spatially filtered in a $150 \mu \mathrm{m}$ diameter pinhole, spectrally filtered by an interference filter (Semrock FF01-420/10) and detected by a photomultiplier in photon-counting mode (Hamamatsu H7828). The overall collection efficiency is on the order of $10^{-3}$. Alternatively, during the alignment process, it is possible to acquire images of the trapped and cooled ions (no spatial filtering) with an electron-multiplier CCD camera (Andor Luca).

\section{Ion Trap Operation}

We observe the stable trapping of ions $\approx 30$ min with Doppler cooling and the ion fluorescence of 1-4 ions. A typical spatially resolved ion fluorescence image of 2 ions is shown in Figure 5a. By fitting the experimental image with two Gaussian spots of the same size, as shown in Figure 5b, we measured an inter-ion distance of $9.5 \pm 0.5 \mu \mathrm{m}$, which corresponds to an ion axial frequency of $305 \pm 26 \mathrm{kHz}$. 1D Plot of the intensity cut line along the inter-ion axial direction of both the ion fluorescence image and its Gaussian fit is shown in Figure 5c for clarification.

We then measured the motional frequencies in the trap using a "tickle" technique ${ }^{[42]}$ coupled to a sequential fluorescence acquisition. In brief, an acquisition sequence is made of thousands of cycles in which first Doppler cooling is applied during roughly $1 \mathrm{~ms}$, then a short (typically $10 \mu \mathrm{s}$ ) pulse of sinusoidal excitation at frequency $f$ is applied to a DC electrode, then the scattered fluorescence photons are acquired during $100 \mu \mathrm{s}$. During the excitation phase, the motional energy of the initially cold ion may increase in a resonant way such that the fluorescence signal acquired in the detection window is affected by Doppler shift. By scanning the frequency $f$, we measured the motional frequencies of a single ion in the trap as a function of trapping parameters (i.e., RF amplitude and DC voltages) with a precision up to $1 \mathrm{kHz}$. Figure 6 shows the average radial frequencies measured as a function of the RF amplitudes (green squares) for a given set of DC voltages (with nominal axial frequency of $300 \mathrm{kHz}$ ). We also plot the theoretical average radial frequency (red diamonds) and the trap depth (black circles), both calculated using an analytical model, ${ }^{[43]}$ to match with the measurement data. Finite element method (FEM) is also used to simulate the ion motion with the designed trap geometry and the radial frequencies are obtained by Fourier transformation of ion position with time, which is given as blue triangles in the plot. 
(a)

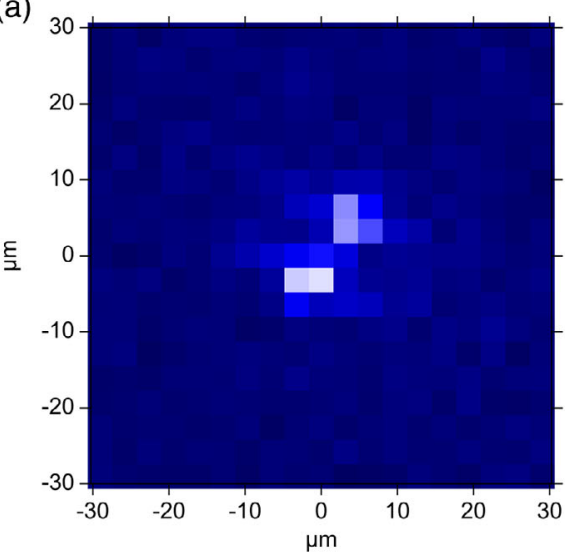

(b)

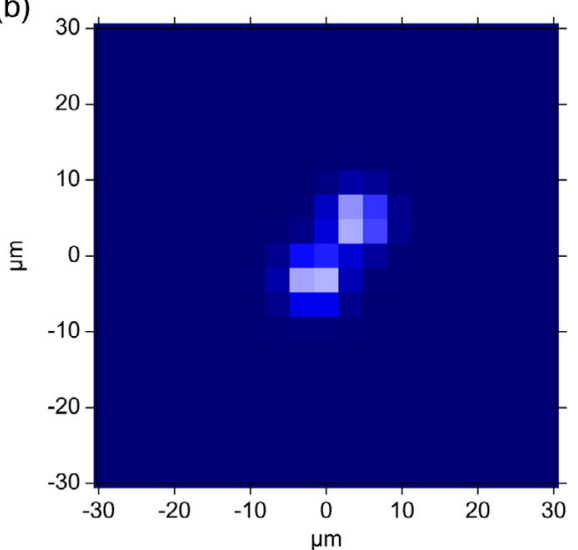

(c)

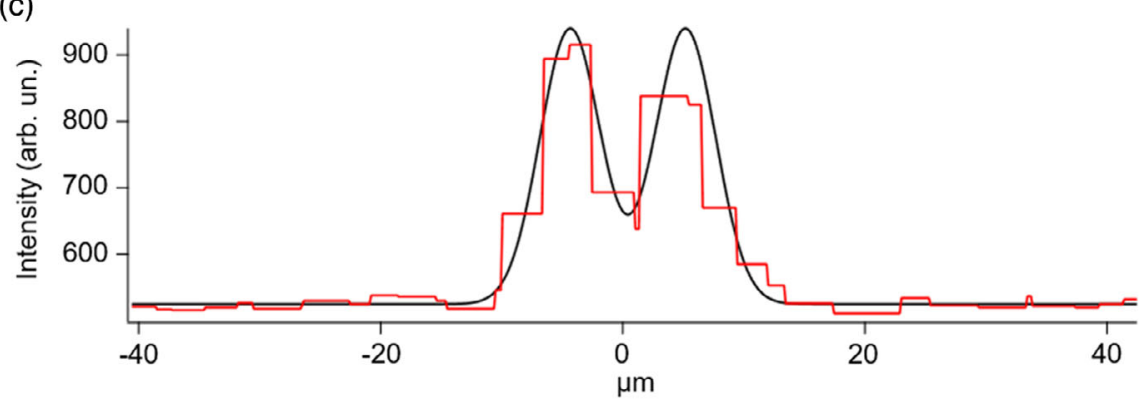

Figure 5. a) Fluorescence image of two trapped ions obtained for a nominal axial frequency of $300 \mathrm{kHz}$. The image (view from above) is acquired with an electron-multiplier CCD camera. b) Best fit of the experimental image with two Gaussian spots of the same size that gives an inter-ion distance of $9.5 \pm 0.5 \mu \mathrm{m}$. This distance corresponds to an axial frequency of $305 \pm 26 \mathrm{kHz}$ ). c) 1D Plot of the intensity cut line along the inter-ion axial direction of both the ion fluorescence image of (a) and its Gaussian fit of (b).

It should be noted that a slight anisotropy with nominal frequency difference between $x$ and $y$ directions on the order of $40 \mathrm{kHz}$ in the plotted range and a tilt of nominal $10^{\circ}$ are imposed by applying asymmetric static voltages in the experiment. An overall good (albeit not perfect) agreement exists between measured, calculated, and simulated frequencies.

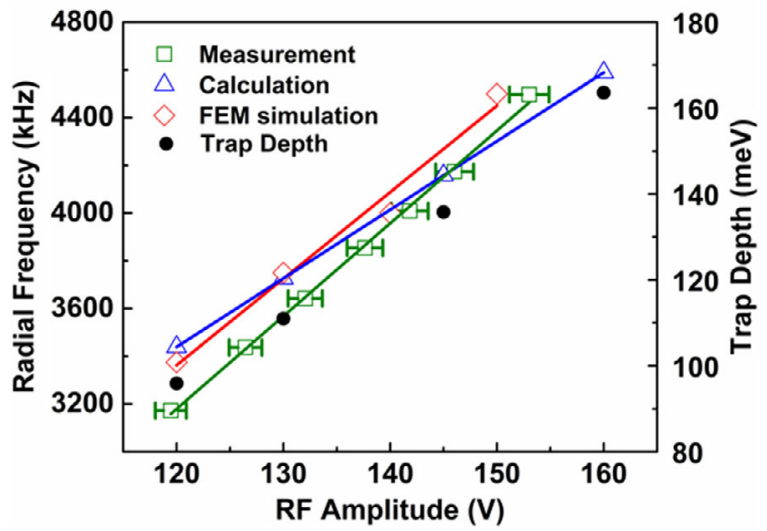

Figure 6. Average radial motional frequency as a function of RF amplitude. The measured data are plotted and matched to analytical calculation and FEM simulated data. The corresponding nominal trap depth (black circles, right axis) is also plotted.
The measured the axial frequency as a function of the nominal axial frequency is shown in Table 2 . This nominal frequency is calculated with the analytical model ${ }^{[43]}$ feed by the electrode geometry and different sets of DC voltages. There is a qualitative agreement between calculations and measurements, with a shift of roughly $100 \mathrm{kHz}$ between the two. Such discrepancies have been observed in other experiments, ${ }^{[38]}$ and are probably due to the approximations inherent to the analytical calculation (e.g., absence of gaps between electrodes). FEM simulation is used to obtain the simulated axial frequencies, which show a better matching to the experimental data than the analytical data. The improvement may be attributed to more accurate geometry (with the defined gaps) built in the FEM model.

Table 2. DC voltage setting and the corresponding measured, nominal, and COMSOL simulated axial frequencies.

\begin{tabular}{lccccccc}
\hline & DC voltages $[\mathrm{V}]$ & & & \multicolumn{3}{c}{ Axial frequency $[\mathrm{kHz}]$} \\
\cline { 1 - 2 } \cline { 6 - 7 } & $V_{\text {EC }}$ & $V_{\text {COMP }}$ & $V_{\text {DCRF }}$ & & Measured & Nominal & Simulated \\
\hline+0.157 & -0.421 & -0.118 & & 265 & 200 & 225 \\
+0.354 & -0.947 & -0.265 & & 405 & 300 & 327 \\
+0.629 & -1.684 & -0.471 & & 500 & 400 & 436 \\
+2.516 & -6.737 & -1.885 & & 895 & 800 & 818 \\
\hline
\end{tabular}


The heating rate of the trap has been characterized using the Doppler recooling method. ${ }^{[4]}$ To implement it, we operate the trap with an axial frequency $\omega_{z} / 2 \pi=490 \mathrm{kHz}$ and radial frequencies around $\omega_{\mathrm{r}} / 2 \pi=3.6 \mathrm{MHz}$. In this situation, the recooling dynamics should be dominated by the temperature of the low-frequency axial mode, even though the cooling laser wavevector has a non-negligible projection along the radial direction. A sequential acquisition first cools the ion during $500 \mathrm{~ms}$, then switches off the cooling laser defining a heating period duration $t_{\mathrm{h}}$, and then switches on again the cooling laser triggering the acquisition of single photons timestamped with their arrival times. The two-level-atom approximation needed for the analysis of the acquired data ${ }^{[44]}$ is well fulfilled by the incoherent repumping approach: we show in Figure 7 (inset) a single-ion fluorescence spectrum that displays a Lorentzian line shape. We record single shot and average histograms of the scattered photons as a function of the emission time for different heating times $t_{\mathrm{h}}$. An example of averaged histogram is shown in Figure 7 with the corresponding fit obtained with the hypothesis of a Maxwell-Boltzmann velocity distribution $\left(T=9.6 \mathrm{~K}\right.$ for $\left.t_{\mathrm{h}}=10 \mathrm{~s}\right)$. From the analysis of all the experimental sets, the heating rate of the trap is evaluated at $960 \pm 50 \mathrm{mK} \mathrm{s}^{-1}$ that corresponds to 40 axial quanta per millisecond. It corresponds to a normalized electric-field spectral noise density, $\omega_{\mathrm{z}} \times S_{\mathrm{E}}\left(\omega_{z}\right)$, of $9 \times 10^{-4}\left(\mathrm{~V} \mathrm{~m}^{-1}\right)^{2}$. This normalized electric-field spectral noise density value lies in between the very dispersed values measured by other groups. ${ }^{[45]}$ We believe that this is certainly not the ultimate heating rate that can be reached with this type of traps as, for example, the electrode surfaces have not been cleaned by ionic bombardment ${ }^{[45]}$ and also the trap is held at $300 \mathrm{~K}$ instead of using a low temperature cryostat.

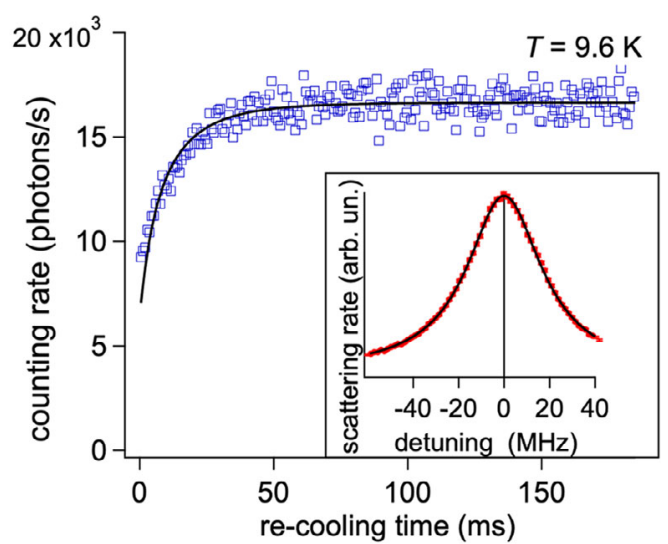

Figure 7. Doppler recooling scattering dynamics of a single ${ }^{88} \mathrm{Sr}^{+}$ion. The counting rate as a function of the recooling time averaged over 100 realizations for a heating period duration $t_{\mathrm{h}}=10 \mathrm{~s}$ is plotted with blue squares. The continuous black curve is the best one-parameter fit that allows us to retrieve a temperature increase $T=9.6 \mathrm{~K}$. Inset: fluorescence spectrum of the ion as a function of the detuning of a probe beam that scans the cooling transition (red circles); the continuous black curve is a Lorentzian fit that shows that the incoherent repumping scheme leads to a very good two-level atom approximation, needed for the validity of the analysis.

\section{Conclusion}

In conclusion, we demonstrated that commercially available Corning glass wafer is a promising low-loss alternative to other wafer substrates for large-scale fabrication of surface ion trap devices. The first tests of a trap designed with a simple five-wire geometry show a very promising trap behavior with respect to RF dissipation, UHV compatibility, and trapping properties. Further systematic studies will allow us to optimize the fabrication process to produce more reliable traps with respect to heating rate suppression.

\section{Acknowledgements}

The authors thank colleagues in Nanyang Nano-fabrication Center and technical staffs in Institute of Microelectronics, $A *$ STAR for their technical supports on trap fabrication and packaging. The work was financially supported by A*STAR Quantum Technology for Engineering (A1685b0005). All authors contributed equally to this work.

\section{Conflict of Interest}

The authors declare no conflict of interest.

\section{Data Availability Statement}

The data that support the findings of this study are available from the corresponding author upon reasonable request.

\section{Keywords}

glass ion traps, large-scale fabrication, laser cooling, radio frequency dissipation

Received: December 1, 2020

Revised: April 23, 2021

Published online: May 19, 2021

[1] D. Leibfried, B. DeMarco, V. Meyer, D. Lucas, M. Barrett, J. Britton, W. M. Itano, B. Jelenković, C. Langer, T. Rosenband, D. J. Wineland, Nature 2003, 422, 412.

[2] C. Monroe, J. Kim, Science 2013, 339, 1164.

[3] B. Lekitsch, S. Weidt, A. G. Fowler, K. Mølmer, S. J. Devitt, C. Wunderlich, W. K. Hensinger, Sci. Adv. 2017, 3, e1601540.

[4] R. Blatt, C. F. Roos, Nat. Phys. 2012, 8, 277.

[5] J. Zhang, G. Pagano, P. W. Hess, A. Kyprianidis, P. Becker, H. Kaplan, A. V. Gorshkov, Z.-X. Gong, C. Monroe, Nature 2017, 551, 601.

[6] K. C. McCormick, J. Keller, S. C. Burd, D. J. Wineland, A. C. Wilson, D. Leibfried, Nature 2019, 572, 86.

[7] V. Tugayé, J.-P. Likforman, S. Guibal, L. Guidoni, Phys. Rev. A 2019, 99, 23412.

[8] J. Chiaverini, R. B. Blakestad, J. Britton, J. D. Jost, C. Langer, D. Leibfried, R. Ozeri, D. J. Wineland, Quantum Inf. Comput. 2005, $5,419$.

[9] S. Seidelin, J. Chiaverini, R. Reichle, J. J. Bollinger, D. Leibfried, J. Britton, J. H. Wesenberg, R. B. Blakestad, R. J. Epstein, D. B. Hume, W. M. Itano, J. D. Jost, C. Langer, R. Ozeri, N. Shiga, D. J. Wineland, Phys. Rev. Lett. 2006, 96, 253003. 
[10] J. M. Amini, H. Uys, J. H. Wesenberg, S. Seidelin, J. Britton, J. J. Bollinger, D. Leibfried, C. Ospelkaus, A. P. Vandevender, D. J. Wineland, New J. Phys. 2010, 12, 33031.

[11] D. L. Moehring, C. Highstrete, D. Stick, K. M. Fortier, R. Haltli, C. Tigges, M. G. Blain, New J. Phys. 2011, 13, 075018.

[12] A. P. VanDevender, Y. Colombe, J. Amini, D. Leibfried, D. J. Wineland, Phys. Rev. Lett. 2010, 105, 023001.

[13] K. K. Mehta, C. D. Bruzewicz, R. McConnell, R. J. Ram, J. M. Sage, J. Chiaverini, Nat. Nanotechnol. 2016, 11, 1066.

[14] T. P. Harty, D. T. C. Allcock, C. J. Ballance, L. Guidoni, H. A. Janacek, N. M. Linke, D. N. Stacey, D. M. Lucas, Phys. Rev. Lett. 2014, 113, 220501.

[15] C. Langer, R. Ozeri, J. D. Jost, J. Chiaverini, B. DeMarco, A. Ben-Kish, R. B. Blakestad, J. Britton, D. B. Hume, W. M. Itano, D. Leibfried, R. Reichle, T. Rosenband, T. Schaetz, P. O. Schmidt, D. J. Wineland, Phys. Rev. Lett. 2005, 95, 60502.

[16] D. T. C. Allcock, T. P. Harty, C. J. Ballance, B. C. Keitch, N. M. Linke, D. N. Stacey, D. M. Lucas, Appl. Phys. Lett. 2013, 102, 044103.

[17] B. Szymanski, R. Dubessy, B. Dubost, S. Guibal, J. P. Likforman, L. Guidoni, Appl. Phys. Lett. 2012, 100, 171110.

[18] D. Stick, K. M. Fortier, R. Haltli, C. Highstrete, D. L. Moehring, C. Tigges, M. G. Blain, arXiv:1008.0990, 2010.

[19] N. D. Guise, S. D. Fallek, K. E. Stevens, K. R. Brown, C. Volin, A. W. Harter, J. M. Amini, R. E. Higashi, S. T. Lu, H. M. Chanhvongsak, T. A. Nguyen, M. S. Marcus, T. R. Ohnstein, D. W. Youngner, J. Appl. Phys. 2015, 117, 174901.

[20] J. Stuart, R. Panock, C. D. Bruzewicz, J. A. Sedlacek, R. McConnell, I. L. Chuang, J. M. Sage, J. Chiaverini, Phys. Rev. Appl. 2019, 11, 24010.

[21] K. K. Mehta, C. D. Bruzewicz, R. McConnell, R. J. Ram, J. M. Sage, J. Chiaverini, Nat. Nanotechnol. 2016, 11, 1066.

[22] D. H. Slichter, V. B. Verma, D. Leibfried, R. P. Mirin, S. W. Nam, D. J. Wineland, Opt. Express 2017, 25, 8705.

[23] K. K. Mehta, A. M. Eltony, C. D. Bruzewicz, I. L. Chuang, R. J. Ram, J. M. Sage, J. Chiaverini, Appl. Phys. Lett. 2014, 105, 44103.

[24] M. Niedermayr, K. Lakhmanskiy, M. Kumph, S. Partel, J. Edlinger, M. Brownnutt, R. Blatt, New J. Phys. 2014, 16, 113068.

[25] A. M. Eltony, S. X. Wang, G. M. Akselrod, P. F. Herskind, I. L. Chuang, Appl. Phys. Lett. 2013, 102, 54106.

[26] M. Töpper, I. Ndip, R. Erxleben, L. Brusberg, N. Nissen, H. Schröder, H. Yamamoto, G. Todt, H. Reichl, in 2010 Proc. 60th Electronic Components and Technology Conf. (ECTC), 2010, pp. 66-73.

[27] M. Lueck, A. Huffman, A. Shorey, in 2015 IEEE 65th Electronic Components and Technology Conf. (ECTC), 2015, pp. 672-677.
[28] H.-K. Li, E. Urban, C. Noel, A. Chuang, Y. Xia, A. Ransford, B. Hemmerling, Y. Wang, T. Li, H. Häffner, X. Zhang, Phys. Rev. Lett. 2017, 118, 53001.

[29] L. Brusberg, N. Schlepple, H. Schröder, in 2011 IEEE 61st Electronic Components and Technology Conference (ECTC), 2011, pp. 805-812.

[30] B. C. Chou, W. Vis, B. Khan, R. Furuya, F. Liu, V. Sundaram, R. Tummala, in 2016 IEEE 66th Electronic Components and Technology Conf. (ECTC), 2016, pp. 497-503.

[31] C. D. Bruzewicz, J. Chiaverini, R. McConnell, J. M. Sage, Appl. Phys. Rev. 2019, 6, 21314.

[32] J. Tao, H. Y. Li, Y. D. Lim, P. Zhao, A. A. Alit Apriyana, L. Guidoni, C. S. Tan, IEEE Trans. Components, Packag. Manuf. Technol. 2020, 10, 679.

[33] S. C. Doret, J. M. Amini, K. Wright, C. Volin, T. Killian, A. Ozakin, D. Denison, H. Hayden, C.-S. Pai, R. E. Slusher, A. W. Harter, New J. Phys. 2012, 14, 73012.

[34] A. A. A. Apriyana, H. Li, P. Zhao, J. Tao, Y. D. Lim, Y. Lin, L. Guidoni, C. S. Tan, IEEE Trans. Components, Packag. Manuf. Technol. 2020, $10,1221$.

[35] L. Floyd, J. Pike, J. Tao, N. Jackson, in Proc. of the 2015 Int. Conf. on Microelectronic Test Structures, IEEE, Piscataway, NJ 2015, pp. 224-229.

[36] M. Brownnutt, V. Letchumanan, G. Wilpers, R. C. Thompson, P. Gill, A. G. Sinclair, Appl. Phys. B 2007, 87, 411.

[37] K. Vant, J. Chiaverini, W. Lybarger, D. Berkeland, arXiv:quant-ph/ $0607055 \mathrm{vl}, 2006$

[38] D. T. C. Allcock, J. A. Sherman, D. N. Stacey, A. H. Burrell, M. J. Curtis, G. Imreh, N. M. Linke, D. J. Szwer, S. C. Webster, A. M. Steane, D. M. Lucas, New J. Phys. 2010, 12, 53026.

[39] D. J. Berkeland, M. G. Boshier, Phys. Rev. A 2002, 65, 33413.

[40] A. A. Madej, L. Marmet, J. E. Bernard, Appl. Phys. B 1998, 67, 229.

[41] B. Dubost, R. Dubessy, B. Szymanski, S. Guibal, J.-P. Likforman, L. Guidoni, Phys. Rev. A 2014, 89, 32504.

[42] M. Vedel, J. Rocher, M. Knoop, F. Vedel, Appl. Phys. B 1998, 66, 191

[43] M. G. House, Phys. Rev. A 2008, 78, 33402.

[44] R. J. Epstein, S. Seidelin, D. Leibfried, J. H. Wesenberg, J. J. Bollinger, J. M. Amini, R. B. Blakestad, J. Britton, J. P. Home, W. M. Itano, J. D. Jost, E. Knill, C. Langer, R. Ozeri, N. Shiga, D. J. Wineland, Phys. Rev. A - At. Mol. Opt. Phys. 2007, 76, 2.

[45] D. A. Hite, Y. Colombe, A. C. Wilson, K. R. Brown, U. Warring, R. Jördens, J. D. Jost, K. S. McKay, D. P. Pappas, D. Leibfried, D. J. Wineland, Phys. Rev. Lett. 2012, 109, 103001. 